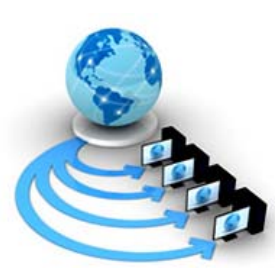

Volume 9, No. 1, January-February 2018

International Journal of Advanced Research in Computer Science

RESEARCH PAPER

\title{
EVALUATION OF MOBILE NETWORK PLANNING STRATEGY WITH ROUTING PROTOCOL SUPPORT
}

\author{
T.R.Muhibur Rahman \\ Research Scholar, \\ JJT University, \\ Jhunjhunu, India
}

\author{
Dr. Nagaraj B.Patil \\ Research Guide, \\ JJT University \\ $\backslash$ Jhunjhunu, India
}

\begin{abstract}
Mobile wireless communication offers development in the last 10 years, motivated by the recognition associated with smart phones as well as tablets. An extensive general opinion within the wireless business anticipates a powerful extension of the pattern for quite some time in the future. The actual progress associated with wireless systems, for example, $4 \mathrm{G}$ as well as Wi-Fi compatibility providing the pervasive Internet entry, the actual visitors' development in the Smartphone-like products offers positioned a growing stress about the mobile network national infrastructure as well as infringed the actual revenue. Because the need is actually growing with the development associated with mobile customers, the actual incumbent heritage national infrastructure has already been phoning to have an update in order to conquer it's current restrictions when it comes to network administration as well as protection. routing within networks is extremely difficult because of a number of features which differentiate all of them through modern communication as well as wireless random networks. This particular document evaluates routing methods which provide the actual network modeling as well as protocols which fulfill QoS needs.
\end{abstract}

Keywords : cellular network, routing protocol, Qos, Network planning, smart phone, Cell, handoff, infrastructure

\section{INTRODUCTION}

Today, many types of research are focused on the making of mobile wireless sensor networks due to their favorable advantage and applications. Allowing the sensors to be mobile will boost the utilization of mobile wireless sensor networks beyond that of static wireless sensor networks. [1]. These types of bottom channels supply the cell using the network protection which may be employed for transmission associated with frequency of voice, information yet others. The cell usually runs on the various groups of frequencies through nearby cells, to prevent disturbance and supply assured support high quality inside every cell [2]. Inside a cellular stereo program, the property region to become provided with stereo support is actually split in to cells, inside a design that depends upon landscape as well as wedding reception features however which could contain approximately hexagonal cell, round or even another normal designs, even though hexagonal cells tend to be traditional.

The most typical instance of the cellular network is really a mobile phone network. The mobile phone is really a transportable telephone that gets or even can make phone calls via a cell location, or even sending structure. Stereo surf is utilized in order to move indicators in order to as well as in the cell phone [3]. Along with CDMA, several CDMA mobile phone models reveal a particular stereo band. The actual indicators tend to be divided using a pseudo sound signal particular in order to every phone. Since the person techniques in one cell to a different, the actual phone creates stereo link along with several cell locations concurrently. This particular is called "soft handoff" simply because, in contrast to along with conventional cellular technologies, there isn't any 1 described stage in which the phone changes towards the new cell [4].
Network routing [5] inside a cellular network handles the actual problems associated with conventional telephone systems, for example, changing as well as phone set up. The majority of cellular network routing problems in various cells could be related to the actual several entry techniques employed for transmission. The place of every mobile phone should be recognized to recycle confirmed music group associated with frequencies in various cells.

The mobile random network (MANET) [6, 7, 8], also called wireless random network or even random wireless network is really a constantly self-configuring, infrastructure-less network associated with mobile products linked wirelessly. Every gadget inside a MANET is actually liberated to proceed individually in a path, and can consequently alter it's linked in order to add products often [9]. The main problem with creating a MANET is actually equipping every gadget in order to constantly keep up with the info necessary to correctly path visitors. This kind of networks might run on their own or even might be attached to the bigger Internet. They might include one or even several and various transceivers in between nodes. This particular leads to a very powerful, autonomous topology.

MANETs tend to be a type of wireless random network (WANET) that always includes a routable networking atmosphere along with a link Coating random network. MANETs contain the peer-to-peer, self-forming, selfhealing network. MANETs circa 2000-2015 usually connect from stereo frequencies (30 MHz - $5 \mathrm{GHz}$ ) [10].

The actual development associated with laptop computers as well as 802. 11/Wi-Fi wireless networking offers created MANETs a well-known investigation subject because of the mid-1990s. Numerous educational documents assess protocols as well as their own capabilities, presuming different examples of the range of motion inside a bounded room, generally along with just about all nodes inside a 
couple of hops of every additional. Various protocols tend to be after that examined depending on steps like the box decrease price, the actual cost to do business launched through the routing protocol, end-to-end box delays, network throughput, the capability to size, and so on.

\section{LITERATURE REVIEW}

The most obvious benefit of MANETs is actually how the network is actually decentralized as well as nodes/devices tend to be mobile, in other words, there isn't any set national infrastructure which supplies the chance with regard to several programs in various places for example environment checking, catastrophe alleviation as well as army communications. Because the earlier 2000s curiosity about MANETs offers significantly elevated that, simply, is actually because of the fact range of motion may enhance network capability [11].

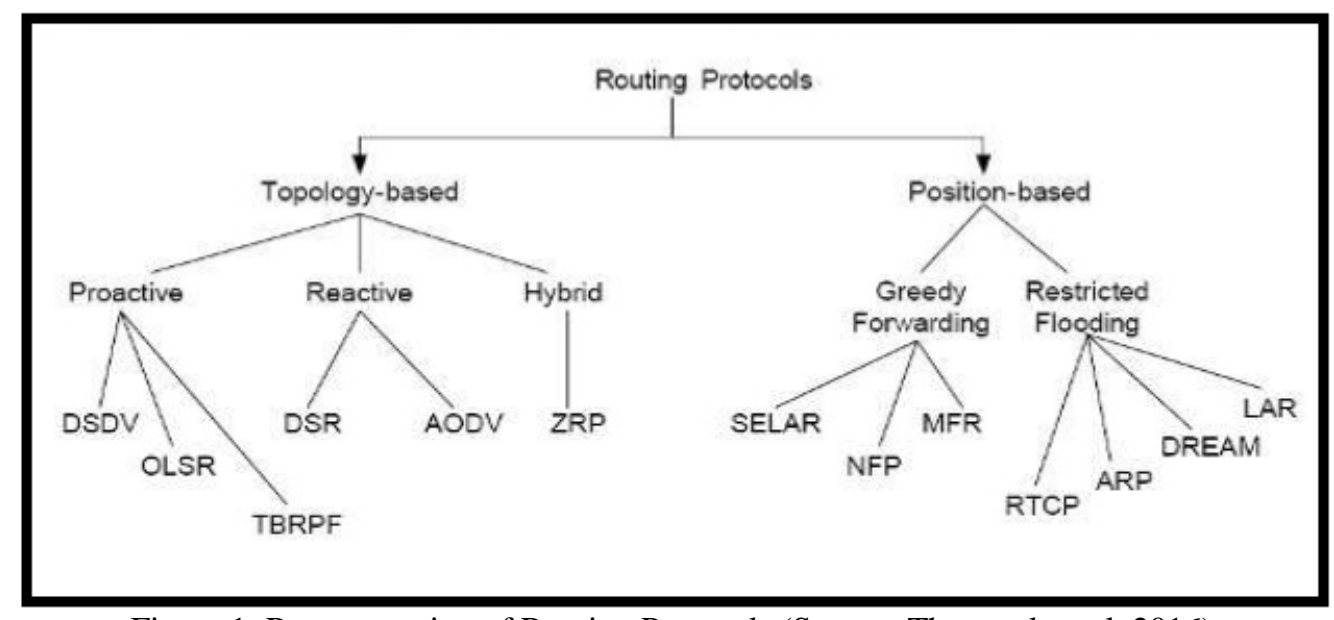

Figure 1: Representation of Routing Protocols (Source: Thangvel et. al, 2016)

One primary benefit to some decentralized network is actually that they're usually better quality compared to centralized networks because of the multi-hop style by which info is actually relayed. For instance, within the cellular network environment, the decrease within protection happens if the bottom train station halts operating; nevertheless, the possibility of just one stage associated with failing inside a MANET is actually decreased considerably because the information may take several pathways. Because the MANET structures change as time goes on it's the actual possible to solve problems, for example, isolation/disconnection in the network [12]. Additional benefits of MANETS more than networks having a set topology consist of versatility, scalability as well as reduce management expenses.

With one of these advantages adhere to a few apparent pull shells within network overall performance. Having a period changing network it's obvious we ought to anticipate variants within network overall performance because of absolutely no set structures. In addition, because network topology decides disturbance and therefore online connectivity, the actual range of motion design associated with products inside the network may affect on network overall performance, perhaps leading to information needing to end up being resent frequently (increased delay) last but not least percentage associated with network assets for example energy continues to be not clear [13]. Lastly, getting a design which precisely signifies human being range of motion although leftover mathematically tractable continues to be a good open up issue because of the big selection of elements which impact this. A few standard models utilized range from the arbitrary stroll, arbitrary waypoint as well as levy flight models.
MANETS may be used with regard to assisting the actual assortment of sensor information with regard to information exploration with regard to a number of programs, for example, polluting of the environment checking as well as various kinds of architectures may be used with regard to this kind of programs. It ought to be mentioned that the crucial attribute associated with this kind of programs is actually which close by sensor nodes checking a good environment function usually sign-up comparable ideals. This sort of information redundancy because of the spatial relationship in between sensor findings creates the actual processes for in-network information aggregation as well as exploration.

Through calculating the actual spatial relationship in between information experienced through various devices, a broad course associated with specific algorithms could be created to build up better spatial information exploration algorithms in addition to better routing methods. Additionally, scientists allow us overall performance models with regard to MANET to use queueing concept. Queueing concept [12] may be the numerical research associated with waiting around outlines, or even lists. The queueing design is actually built to ensure that line measures as well as waiting around period could be forecasted. Queueing concept is usually regarded as the department associated with procedures investigation since the solutions are frequently utilized when creating company choices concerning the assets required giving a support.

The actual $\mathrm{M} / \mathrm{M} / 1$ line is really a easy design the place where a solitary server acts work which appear based on the Poisson procedure and also have tremendously dispersed support needs. Within an M/G/1 line, the actual Grams means common as well as signifies a good irrelevant likelihood submission. The actual M/G/1 design had been 
resolved through Felix Pollaczek within 1930, [12] a solution later on recast within probabilistic conditions referred to as Pollaczek-Khinchine method [13].

Networks associated with clients are also looked into; Kelly networks exactly where clients associated with various courses encounter various concern amounts from various support nodes. Another kind of network tends to be Gnetworks very first suggested by Gelenbe. These types of networks don't presume rapid period distributions such as the traditional Fitzgibbons network.

Within under the radar period networks exactly where there's a restriction which supports nodes could be energetic anytime, the actual max-weight arranging formula selects something to plan to provide optimum throughput in case that evey work appointments merely a solitary support node. Within the much more common situation exactly where work may go to several nodes, backpressure routing provides optimum throughput. The network scheduler should select a queuing formula, which impacts the actual features of the bigger network.

\section{RESEARCH METHODOLOGY}

Every area is the topics of extreme analysis pursuits offering powerful alternatives to these kind of issues. Particularly, a large body of research aimed at developing a effective wireless mesh central source by discovering state-of-the-art multiradio, multi-channel, and multi-rate features, applying heterogeneous cellular technology along with kinds of antennas. In present research we compare the existing network planning strategy and evaluate the new one.

\subsection{Traditional Network Planning}

The normal strategy is actually which actual packets from the reside software tend to be delivered to the actual emulation server. The actual box will get 'modulated' right into a simulation box. The actual Simulation box will get demodulated into the actual box following going through results associated with a reduction, hold off, jitter etc. therefore moving these types of network results to the actual box [14]. Therefore it's as if the actual box ran via an actual network however in actuality this ran with the simulated network. Emulation is actually popular in the style phase with regard to validating communication networks just before deployment.

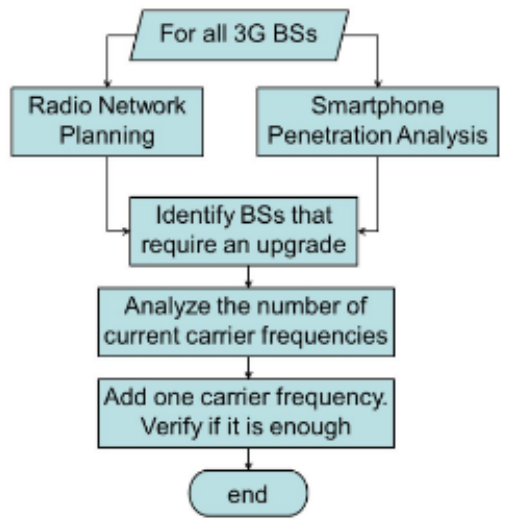

Figure 2: Traditional Network planning Flowchart (Source: Caroline Jennings, 2015)

Additional, network preparing as well as style is definitely an iterative procedure, covering topological style, network- synthesis, as well as network-realization, and it is targeted at making certain a new telecommunications network or even support fulfills the requirements from the customer as well as owner. The procedure could be customized based on every new network or even support. A conventional network preparing strategy within the framework associated with company choices entails 5 levels associated with preparing because require evaluation as well as source evaluation, short-term network preparing, THIS source, long-term as well as medium-term network preparing, Procedures as well as upkeep [15]. All these levels include programs with regard to various period horizons, the i.e. company preparing to coat decides the look how the owner should carry out to ensure the actual network may carry out because necessary for it's meant life-span. The Operations and Maintenance layer, however, examines how the network will run on a day-to-day basis.

\subsection{New Network Planning Approach}

Planning for a new support entails applying for the new program over the very first 4 levels from the OSI Research Design. Options should be designed for the actual protocols as well as transmission technologies.

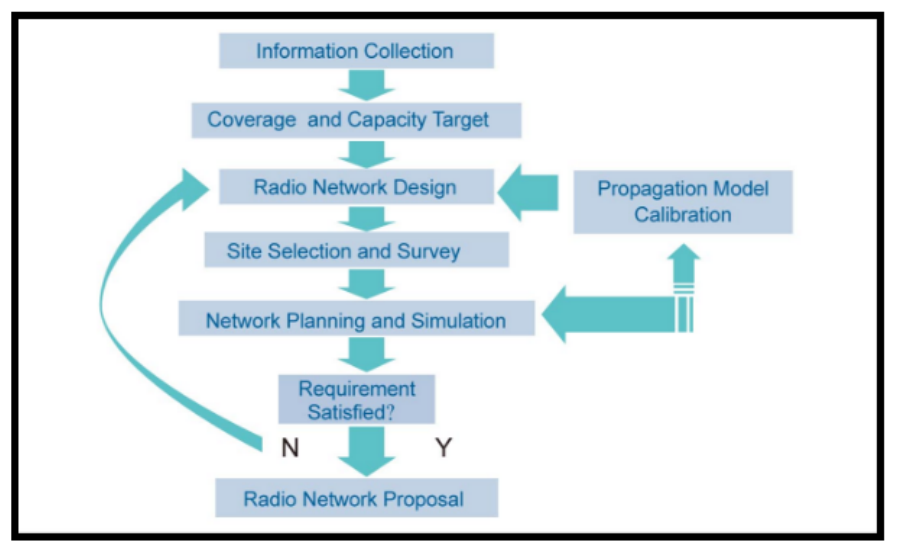

Figure 3: ZTE Network Planning Procedure (Source: ZTE Corp)

The actual network preparing procedure starts using the purchase associated with exterior info. Including predictions associated with the way, the new support may run, the actual financial info regarding expenses and also the specialized information on the actual network's abilities.

\section{CONCLUSION}

Contemporary mobile phone networks make use of cells simply because stereo frequencies really are a restricted, discussed source. cell-sites, as well as mobile phone models, alter the rate of recurrence below pc manage as well as make use of reduced energy transmitters so the generally restricted quantity of stereo frequencies could be concurrently utilized by numerous callers along with much less disturbance. Since the phone techniques in one cell region to a different cell whilst the phone is within improvement, the actual mobile train station may visit a new band to add in order to so as to not decrease the phone call. As soon as a new band is located, the actual network may order the actual mobile device to change towards the new frequency band as well as simultaneously change the phone call on to the new band. Therefore, there's require associated 
with new network preparing strategy that fits for those rate of recurrence rings regardless of protocols.

\section{REFERENCES:}

[1] Udagepola, K. P. "Mobile Sensor Networks and Robotics." Internet of Things and Big Data Analytics Toward Next-Generation Intelligence. Springer, Cham, 2018. 21-46.

[2] Kontik, Mehmet, and Sinem Coleri Ergen. "Distributed Medium Access Control Protocol for Successive Interference Cancellation-Based Wireless Ad Hoc Networks." IEEE Communications Letters 21.2 (2017): 354-357.

[3] Narayana, V. Lakshman, and C. R. Bharathi. "IDENTITY BASED CRYPTOGRAPHY FOR MOBILE AD HOC NETWORKS." Journal of Theoretical and Applied Information Technology 95.5 (2017): 1173.

[4] Gupta, Riten, et al. "Routing in Mobile Ad-Hoc Networks using Social Tie Strengths and Mobility Plans." Wireless Communications and Networking Conference (WCNC), 2017 IEEE. IEEE, 2017.

[5] Zhang, Xin Ming, et al. "Interference-based topology control algorithm for delay-constrained mobile ad hoc networks." IEEE Transactions on Mobile Computing 14.4 (2015): 742-754.

[6] Wang, Yanwei, et al. "A mean field game theoretic approach for security enhancements in mobile ad hoc networks." IEEE Transactions on wireless communications 13.3 (2014): 16161627.
[7] Al-Sultan, Saif, et al. "A comprehensive survey on vehicular ad hoc network." Journal of network and computer applications 37 (2014): 380-392.

[8] Karaoglu, Bora, and Wendi Heinzelman. "Cooperative load balancing and dynamic channel allocation for cluster-based mobile ad hoc networks." IEEE transactions on mobile computing 14.5 (2015): 951-963.

[9] Chen, Ray, et al. "Trust management in mobile ad hoc networks for bias minimization and application performance maximization." Ad Hoc Networks 19 (2014): 59-74.

[10] Zhang, Daqiang, et al. "BASA: Building mobile Ad-Hoc social networks on top of android." IEEE Network 28.1 (2014): 4-9.

[11] Veni, R. Marutha, and R. Latha. "Mobile ad hoc network." international Journal of Science and Research (iJSR) 2.4 (2013).

[12] Akbani, Rehan, Turgay Korkmaz, and G. V. S. Raju. "Mobile ad-hoc networks security." Recent Advances in Computer Science and Information Engineering. Springer Berlin Heidelberg, 2012. 659-666.

[13] Zhang, Xin Ming, et al. "A neighbor coverage-based probabilistic rebroadcast for reducing routing overhead in mobile ad hoc networks." IEEE transactions on mobile computing 12.3 (2013): 424-433.

[14] Huang, Kaibin, et al. "Spatial interference cancellation for multiantenna mobile ad hoc networks." IEEE Transactions on Information Theory 58.3 (2012): 1660-1676.

[15] Hinds, Alex, et al. "A review of routing protocols for mobile ad-hoc networks (manet)." International journal of information and education technology 3.1 (2013). 